\title{
A new fuzzy MCDA framework for make-or-buy decisions: A case study of aerospace industry
}

\author{
Mohsen Cheshmberah ${ }^{a^{*}}$, Ahmad Makui, Seyed Mohammad Seyedhoseini ${ }^{*}$, and Ali Najmi
}

${ }^{a}$ Department of Industrial Engineering, Iran University of Science \& Technology, Tehran, Iran

\begin{tabular}{l}
\hline A R T I C L E I N F O \\
\hline Article history: \\
Received October 202010 \\
Received in revised form \\
22 February 2011 \\
Accepted 23 February 2011 \\
Available online \\
25 February 2011 \\
\hline Keywords: \\
Make-or-buy \\
Outsourcing \\
PROMETHEE \\
Aerospace industry \\
Decision making
\end{tabular}

\section{A B S T R A C T}

\begin{abstract}
One of the primary managerial decisions for manufacturing units is to find out which activity must be outsourced. A good outsourcing decision is normally involved with different criteria such as core competencies, cost, technology and relative capability, risk, etc. In this paper, we present a multi criteria decision-making method to find a suitable solution for outsourcing activities called preference ranking organization method for enrichment evaluations (PROMETHEE). The proposed model of this paper uses fuzzy numbers to determine the relative importance of different criteria and it is implemented for a real-world case study of aerospace industry.
\end{abstract}

\section{Introduction}

Outsourcing is a common practice among the organizations and is a major element in business strategy (kremic, et al., 2006). It has recognized as an area of strategic importance (Jenings, 1997). Naturally all organizations will expect different benefits from outsourcing. Developing the use of outsourcing has numerous advantages and benefits such as increase focus on core functions, access to economies of scale and cost savings, transfer fixed costs to variable, access to latest technology and external skills and talent, greater flexibility and increased speed, the ability to focus on the specialized activities, quality improvement, reduction in overheads, and etc. (Quinn, et al., 1994; Jenings, 1997; kremic et al., 2006). There are some risks associated with outsourcing. Many potential risks are hidden costs, less flexibility, poor selection of partner, poor contract, loss of core competencies, loss of knowledge and skills, loss of control, power-man shift to supplier, poor performance of supplier, poor morale in employees, competitor creation, security issues, skill erosion, loss of control over a supplier, and etc (Quinn, et al., 1994; kremic, et al., 2006).

The primary question is to choose appropriate activities for outsourcing which leads to a make-or-buy strategy (Platt et al., 2002). During the past few years, there have been tremendous efforts on make-

* Corresponding author. Tel: +982173227000

E-mail addresses: mcheshmberah66@gmail.com (M. Cheshmberah ) Seyyedmohammad@iust.ac.ir (S.M. Seyedhosseini) 
or-buy decisions (McIvor \& Humphreys, 2000). Make-or-buy decisions are normally considered as strategic decisions (Canez, 2000; McIvor et al., 1997). However, there are other factors influencing the make-or-buy decisions such as quality, delivery performance, cost, flexibility and innovation (Leong et al., 1990). A good decision on buy or make could be made through investigating the available resources. Cox (1997), Venkatesan (1992) and McIvor, et al. (1997) analyzed make-or-buy decision-making based on resource viewpoint where the decision is made by focusing on the firm's resources. The purpose of this work is to retain core activities and outsource only non-strategic activities. There are other works to concentrate on technology for outsourcing activities (Probert, 1997). Welch and Nayak(1992) introduced three factors namely "maturity level of process technology", "competitors' process technology position", and "importance of process technology" as basis for a decision matrix called "strategic sourcing model".

Quinn and Hilmer (1994) proposed "core competency" and "strategic vulnerability" as the make-orbuy decision making criteria. Fine and Whitney (1996) considered capability, manufacturing competitiveness, and technology as the outsourcing reasons. Jennings (1997) provided a framework for evaluating the outsourcing decisions with a set of strategic guidelines based on several areas: business environment, cost, supplier relationships, technology, and capability. Fill and Visser (2000) presented a conceptual framework for make-or-buy decision making consisting of contextual factors, strategy \& structure, and transaction costs. McIvor and Humphreys (2000) discussed a computerbased system using "case-based reasoning" approach for make-or-buy decisions. Insinga and Werle (2000) applied "potential of an activity on yield competitive advantage" and "Internal capability to perform an activity in comparison with competitors" in a two-dimensional decision matrix.

Canez et al. (2000) and Platt et al. (2002) applied technology \& manufacturing processes, cost, supply chain management \& logistics, and support systems as influencing areas in make-or-buy decision making and developed a set of criteria related to each area. From capacity prospective, outsourcing can be divided into capacity vs. non-capacity outsourcing. Capacity outsourcing refers to activities which are executed internally (in-house) and temporarily, internal capacity is not enough for demand responding because of demand fluctuations. The second category concerns the outsourcing of activities, which are no longer pursuit by the firm itself (Fill \& Visser, 2000). Moreover, outsourcing may be categorized to full, selected, and everything-in-between outsourcing discussion that refers to assignment of one, few or many (total) of activities (functions) to suppliers (Fill \& Visser, 2000).

Pandey and Bansal (2004) considered criticality, stability, and simplicity as the factors for selecting activities to outsource (IT outsourcing environment). Water and Peet (2006) inspiration from Canez et al.(2000) and Platt et al. (2002) led their studies and applied engineering and R\&D, human resources, finance, supply chain management \& logistics, support systems, and technology \& manufacturing as the important areas for outsourcing decision-making. Kremic et al. (2006) proposed strategy, cost, function characteristics, and environment as factors, which may affect outsourcing decisions. Mantel et al. (2006) applied the criteria originally developed by Quinn and Hilmer (1994) to develop operational sub-criteria. Wang and Yang (2007) discussed six factors including: economic, resource, strategy, risk, management, and quality for information systems outsourcing decisionmaking. Chopra and Meindl (2007) considered "demand scale", "demand uncertainty", and "specificity of assets" as the factors influencing "propensity to outsource" and Hwang et al. (2007) applied manufacturing technology, outsourcing risk, and managerial, financial, and operational conditions as the items for evaluation of a make-or-buy decision-making problem. McIvor (2010) discussed about usefulness of the resource-based theory in the manufacturing outsourcing decisions. He presented a matrix as the decision framework based on "competitive advantage" and "capability position".

The make-or-buy decision making is an important issue because these decisions determine the boundaries of a firm (Lonsdale \& Cox, 2000). Regarding to the complexity and the nature of outsourcing decision-making, this problem can be considered as a multi-criteria problem (Canez et al., 2000, Probert et al., 2001, Platt et al., 2002, Mantel et al., 2006, Henny \& Peet, 2006, Hwang et al., 2007, Wang \& Yang, 2007). Moreover, in some firms, the models are often derived based on idealized scenarios without empirical support, and therefore do not necessarily reflect how the firm 
actually arrives at decisions (Moses \& Ahlstrom, 2008). The organization of this paper is as follows. The problem statement is explained in section 2. Section 3 presents a conceptual framework for make-or-buy decision making. Finally, the application of the decision-making framework for a realworld case study using fuzzy PROMETHEE method is presented.

\section{Problem statement}

The proposed study of this paper uses a multi criteria decision making models to find efficient strategy to decide which activities must be ordered and which ones need to be built. We implement the proposed model for a real-world case study of aerospace industry. There are two primary assumptions associated with our model. The first assumption assumes that there is no leakage for strategic information. The second assumption insures that there is precise data so the judgments are based on fuzzy concepts and the verbal judgments of experts are used.

Consequently, for outsourcing decision making of the case study of this paper, a framework is required to consider the risk of leakage information and a multi criteria model based on linguistic judgments of experts.

\section{Developing the decision-making framework}

The proposed model of this paper consists of four different dimensions of core competencies, information security, technological, economic. Fig. 1 shows the details of all components.

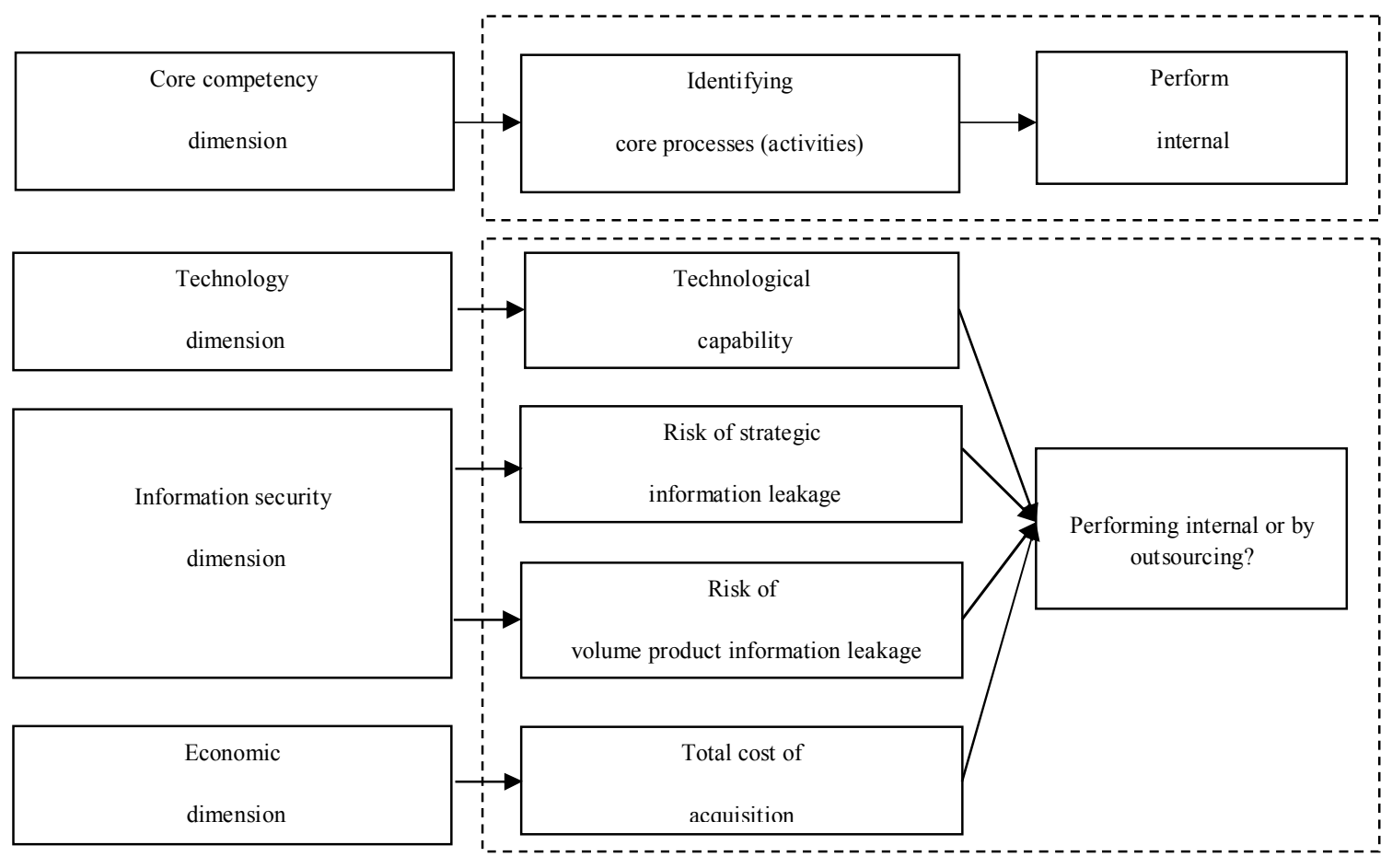

Fig. 1. Outsourcing decision making framework for the studied firm

As we can see from Fig. 1, each dimension itself has different components and a detailed analysis of all these components lead to an appropriate decision as to whether we should buy or build a part.

\subsection{Core competencies dimension}

Prahalad and Hamel (1990) argued that core competencies in a company have, at least, three attributes:

- they provide a potential approach to various markets, 
- play an important role in customers intuition and

- are difficult to imitate (by competitors).

Note that all goods and services, which are considered core competencies, must be built, internally (Venkatesan, 1992; McIvor, et al., 1997; Arnold, 2000).

\subsection{Information security (leakage risk) dimension}

One important issue in outsourcing decision-making is that how information security can be realized (Kajava, et al., 2006). The risks of information leakage can be divided into two categories as follows,

- Risk of strategic information leakage

- Risk of volume product information leakage

Information of sales, strategic directions and policies, target (niche) markets and other issues are examples of strategic information where they may have some confidential information. The disclosure of strategic secrets may result in competitive damage (Hoecht \& Trott, 2006) and irreparable losses to organizations. Second category of information leakage is the volume information of products; sometimes knowing the accurate quantity of a product may be important for the competitors. Outsourcing an activity of a product may disclose this kind of information. If this kind of information is important and the impact of related risk is high, it is important to protect them as much as possible.

\subsection{Technological dimension}

The third important factor influencing outsourcing activities is the technological capability to perform processes or activities. There are cases where the level of technology inside a firm is higher than the other firms, which are acting as suppliers. There are literally studies that indicate that technological capability play an important role on outsourcing decisions (Canez et al., 2000; Fill \& Visser, 2000; Henny \& Peet, 2006; Hwang, et al., 2007; Jennings, 1997; Welch \& Nayak, 1992, Probert, 1997; Platts et al., 2002; Wang \& Yang, 2007). Technological capabilities generally follow the life cycle of technology (Bin, 2000), the maturity level and the competitors (Welch \& Nayak, 1992). Obviously, specific level of technological capability is required to meet the minimum requirements for the product quality. However, the supplier with higher levels of technological capabilities is preferred (Fine \& Whitney, 1996).

\subsection{Economic dimension}

Fourth outsourcing decision-making dimension in our study is economic dimension. There are some other studies suggesting total cost and cost saving as major criteria for economic dimension of outsourcing decision-making (Arnold, 2000; Canez et al., 2000; Henny \& Peet, 2006; Hwang et al., 2007; Jennings, 1997; Platts, et al., 2002; Kremic et al., 2006; Wang \& Yang, 2007). In this study, we select "total cost" as the economic criteria. By adopting Porter's value chain, the range of processes in this article is manufacturing processes (or activities) associated with a specific product called "operations" (Porter \& Miller, 1985).

\section{The real case of framework application}

Consider an activity $A-L 112$ of product $A$ where we want to decide whether we should buy or make it inside a firm. The part contains a high level of confidential information and, at the same time, it needs a high level of technology for production, which limits the number of potential suppliers to only two or three firms. The team of outsourcing decision-making has already specified that A-L112 is not core activity. Therefore, in order to make an outsourcing decision for this activity, other criteria i.e. technological capability, risk of strategic information leakage, the risk level of the product information leakage, and total cost must be considered (Fig. 1). As we explained earlier, we do not know the exact figures of the information. Therefore, we use fuzzy numbers to identify them.

\subsection{Fuzzy data utilization}


The use of fuzzy numbers for the evaluation of each alternative is adequate and important, since its usage allows a closer look at the reality of the problem, obtaining a more realistic ranking.

The data for the performance of each alternative based on criterion, the data of weight of criteria have been obtained through discussion with group of experts for our case study, and the data have been introduced as linguistic variables. This comes from the fact that in most cases the input data cannot be defined within a reasonable degree of accuracy. This imprecision is sometimes treated as a probability, where a stochastic approach is applicable. However, the happening of this fuzzily described situation is certain and the fuzzy set theory is considered more appropriate. Bellman and Zadeh (1970) discussed the characteristics of fuzziness and randomness in decision-making.

Fuzzy numbers are presented, according to Wang and Chang (2007), in terms of triangular numbers $x=\left(a^{l}, a^{m}, a^{u}\right)$. When the variable $x$ has the value $a^{m}$ it is certain to belong to the specific class and its membership function $f(x)=1$. If it has values smaller than $a^{l}$ and bigger than $a^{u}$ it does not belong in the specific class and $f(x)=0$. In the interval between $a^{l}<a^{m}<a^{u}$ there is a 'grade of membership' of $x$ in the specific class associated with a number between 0 and 1 .

Table 1 shows linguistic ratings of four alternatives in decision-making criteria for $A$-L112. In-house alternative is performing activity $A-L 112$ within the firm and Out-A, Out-B and Out-C are three potential suppliers. The ratings for alternatives consist of very low (VL), low (L), medium low (ML), medium (M), medium high (MH), high (H), and very high (VH) (Zimmerman, 1991; Bottani \& Rizzi, 2006). Fig. 2 shows the fuzzy numbers in terms of different levels of uncertainties. In addition, the weights of criteria are determined based on the feedback we have from a team of outsource decisionmaking experts of company. Table 1 shows the details of the results of our survey.

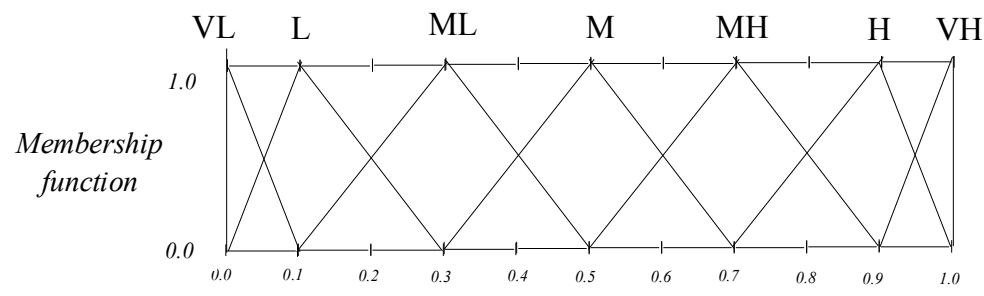

Fig. 1. The fuzzy numbers for alternatives

Table 1

Performance of four alternative scenarios for the outsourcing

\begin{tabular}{ccccc}
\hline Risk of strategic & $\begin{array}{c}\text { Risk of volume product } \\
\text { information }\end{array}$ & information leakage & Total cost & $\begin{array}{c}\text { Technological } \\
\text { capability }\end{array}$ \\
\hline Weights & 0.3 & 0.25 & 0.15 & 0.3 \\
\hline In-house & Very Low & Very Low & High & Medium \\
Out-A & Low & Low & Medium Low & Medium \\
Out-B & Medium Low & Medium & Medium Low & Medium High \\
Out-C & Medium & Medium & Low & High \\
\hline
\end{tabular}

\subsection{Application of PROMETHEE II method}

PROMETHEE is an outranking method, which was initially introduced by Brans et al. (1984), Brans and Vincke (1985), and Brans et al. (1986). It is well adapted to the problems where a finite set of alternatives are to be ranked according to several, sometimes conflicting criteria (Albadvi et al., 2007; Dag deviren, 2008). The evaluation is the starting point of PROMETHEE method where alternatives are evaluated with respect to different criteria.

Adaptations of the PROMETHEE method to use fuzzy numbers in the evaluations of alternatives required the following steps: 
Step 1: Assuming the evaluations of alternatives under a given criterion $j$ as triangular fuzzy numbers, the difference $d(a, b)$ between the evaluations of two alternatives $a$ and $b$, will be a triangular fuzzy number $\left(a^{l}, a^{m}, a^{u}\right)$, represented as a rating of (Dubois \& Prade, 1978). Table 2 shows the difference $d(a, b)$ among various alternatives.

Table 2

The difference $d(a, b)$ between alternatives under total cost criterion

\begin{tabular}{ccc}
\hline Alternative a & Alternative $\mathbf{b}$ & $\boldsymbol{d}(\boldsymbol{a}, \boldsymbol{b})$ \\
\hline In house & Out-A & $(-0.9,-0.6,-0.2)$ \\
In house & Out-B & $(-0.9,-0.6,-0.2)$ \\
In house & Out-C & $(-1,-0.8,-0.4)$ \\
Out-A & In house & $(0.2,0.6,0.9)$ \\
Out-A & Out-B & $(-0.4,0,0.4)$ \\
Out-A & Out-C & $(-0.5,-0.2,0.2)$ \\
Out-B & In house & $(0.2,0.6,0.9)$ \\
Out-B & Out-A & $(-0.4,0,0.4)$ \\
Out-B & Out-C & $(-0.5,-0.2,0.2)$ \\
Out-C & In house & $(0.4,0.8,1)$ \\
Out-C & Out-A & $(-0.2,0.2,0.5)$ \\
Out-C & Out-B & $(-0.2,0.2,0.5)$ \\
\hline
\end{tabular}

STEP 2: The function of preferences for each criterion depends on the type of problem, one can consider that, in most cases, the type $\mathrm{V}$ preference function (with linear zone of indifference) is one of the most adequate one (BRANS et al. 1986). The preferably function or generalized criterion represents the behavior or attitude of the decision maker regarding the differences among options for a given $j$ criterion.

STEP 3: Constructing the fuzzy preference function, the preference function $\widetilde{P}_{J}(\mathrm{a}, \mathrm{b})$ can be defined as follow,

$$
\tilde{P}_{j}(a, b)=\left\{\begin{array}{ll}
0 & , \tilde{x}_{a j} \leq \tilde{x}_{b j} \\
\tilde{x}_{a j}-\tilde{x}_{b j} & , \tilde{x}_{a j}>\tilde{x}_{b j}
\end{array} j=1,2, \ldots, k\right.
$$

where the preference function $\widetilde{P}_{j}(a, b)$ means the outranking intensity that $a$ is superior to $b$.

The outranking relationship construct from the pair-wise comparison of alternatives.

$$
\left\{\begin{array}{l}
\tilde{x}_{a j}>\tilde{x}_{b j} \Leftrightarrow a P b \quad(\text { a outranks } b) \\
\tilde{x}_{a j}=\tilde{x}_{b j} \Leftrightarrow a I b \quad(\text { a is indifferent } b)
\end{array}\right.
$$

STEP 4: Define the multi-criteria preference index to decide the valued outranking relationship. The multi-criteria preference index is expressed as follows,

$$
\tilde{\pi}(a, b)=\frac{\sum_{j=1}^{k} w_{j} \tilde{P}_{j}(a, b)}{\sum_{j=1}^{k} w_{j}}
$$

where, $W_{j}$ is the weight assigned to the criterion $j$ and $\tilde{P}_{j}(a, b)$ is given as $\tilde{P}_{j}\left[d_{j}(a, b)\right]$. In addition, $\tilde{P}_{j}(a, b)$ refers to the value of the preference function according to the difference between the evaluations of the alternatives $a$ and $b$ on the criterion $j . \tilde{\pi}(a, b)$ represents the intensity of preference of the decision maker of alternative " $a$ " over action " $b$ " when considering all the criteria, simultaneously.

STEP 5: Calculate the flows to preorder the alternatives. The leaving flow and entering flow will be fuzzy numbers according to Eq. (4) and Eq. (5), respectively. Results of calculating the leaving and entering flows are given in the second and third columns of Table 3. 


$$
\widetilde{\Phi}_{(a)}^{+}=\sum_{\substack{b=1 \\ b \neq a}}^{n} \tilde{\pi}(a, b)
$$

$$
\widetilde{\Phi}_{(a)}^{-}=\sum_{\substack{b=1 \\ b \neq a}}^{n} \tilde{\pi}(b, a)
$$

\section{Table 3}

The rank of alternatives

\begin{tabular}{cccccc}
\hline Alternatives & leaving flow $\left(\Phi^{+}{ }_{\mathrm{a})}\right)$ & entering flow $\left(\Phi^{-}{ }_{(\mathrm{a})}\right)$ & Net flow $\left(\Phi^{-}(\mathrm{a})\right)$ & Yager index & Rank \\
\hline In house & $(0.16,0.545,1.055)$ & $(0.12,0.48,0.985)$ & $(-0.825,0.065,0.935)$ & 0.058 & 2 \\
Out-A & $(0.03,0.47,1.17)$ & $(0,0.265,0.87)$ & $(-0.82,0.205,1.17)$ & 0.178 & 1 \\
Out-B & $(0.03,0.27,0.985)$ & $(0.05,0.465,1.215)$ & $(-1.185,-0.195,0.935)$ & -0.148 & 4 \\
Out-C & $(0.06,0.48,1.03)$ & $(0.11,0.555,1.17)$ & $(-1.11,-0.075,92)$ & -0.088 & 3 \\
\hline
\end{tabular}

Step 6: The net flow is computed based on the fact that the higher the leaving flow and the lower the entering flow, the better the action. The net flow is also a fuzzy number obtained through the difference between leaving and entering flows.

$$
\widetilde{\Phi}(a)=\widetilde{\Phi}^{+}(a)-\widetilde{\Phi}^{-}(a)
$$

STEP 7: The results of the calculations of leaving flow and entering flow are in the form of fuzzy numbers and the main problem is calculations the net flow. In the present work, the index proposed by Yager (1981) has been used, which corresponds to a 'weight average' of the fuzzy number. It is determined by the center of weight of the surface representing its membership function.

$$
x_{\text {defuz }}=\frac{1}{3}\left(a^{l}+a^{m}+a^{u}\right)
$$

The ranking by the PROMETHEE II method are shown in sixth column of Table 3.

\section{Conclusion}

In this paper, an outsourcing decision making framework based on the conditions of a manufacturing firm has been developed. The firm manufactures parts and subassemblies for some products in aerospace industries. The core competency, technological capability, risk of strategic information leakage, risk of the product leakage information, and total cost applied as the criteria for outsourcing decision-making framework. The framework was implemented in outsourcing decision-making for a real-world case study of aerospace industry. Since we did not have precise information about the case-study we decided to use fuzzy numbers and PROMETHEE as a outranking method was applied to ranking alternatives.

\section{References}

Albadvi, A., Chaharsooghi, S. K., \& Esfahanipour, A. (2007). Decision making in stock trading: An application of PROMETHEE. European Journal of Operational Research, 177, 673-683.

Arnold, U. (2000). New dimensions of outsourcing: a combination of transaction cost economics and the core competencies concept. European Journal of Purchasing Supply Management, 6, 23-29.

Bellman, R., \& Zadeh, L.A. (1970). Decision making in fuzzy environment. Management Science, 17, 141 164.

Brans, J. P., Mareschal, B., Vinke, P. (1986). How to select and how to rank projects: The PROMETHEE method. European Journal of Operational Research, 24, 228-238.

Brans, J. P., Mareschal, B., \& Vincke, P. P. (1984). PROMETHEE: A new family of outranking methods in $M C D M$. In J. P. Brans (Ed.), Operational Research (477-490). Amsterdam: North-Holland.

Brans, J. P., \& Vincle, P. (1985). A preference ranking organization method. Management Science, 31, 647656.

Canez, L., Platt, K.W., \& Probert, D.R. (2000). Developing a framework for make-or-buy decisions. International Journal of Operations Production Management, 20(11), 1313-30. 
Canez, L. (2000). Industrial make-or-buy decisions: Developing a framework and a practical process. Ph.D. Thesis, University of Cambridge, Cambridge, United Kingdom.

Dag deviren, M. (2008). Decision making in equipment selection: an integrated approach with AHP and PROMETHEE. Journal of Intelligent Manufacturing, 19, 397-406.

Dubois, D. \& Prade, H. (1978). Operations on Fuzzy Numbers. International Journal of Systems Science, 9(6), 613-626.

Guo, B. (2000). Life cycle view of firm's core competence: Chinese manufacturing industry as a case. IEEE International Conference, 363-367.

Fill, C., \& Visser, E. (2000). The outsourcing dilemma: a composite approach to the make or buy decision, Management Decision, 38(1), 43-50.

Fine, C.H., Whitney, D.E. (1996). Is the make-buy decision process a core competence?, MIT Center for Technology, Policy, and Industrial Development, Cambridge, working paper no. 3875-96

Hoecht, A., \& Trott, P. (2006). Outsourcing, information leakage and the risk of losing technology-based competencies. European Business Review, 18(5), 395-412.

Hwang, H.S., Ko, W.H., \& Goan, M. J. (2007). Web-based multi-attribute analysis model for make-or-buy decisions, Mathematical Computers Modeling, 46, 1081-1090.

Jennings, D. (1997). Strategic guidelines for outsourcing decisions. Strategic Change, 6, 85-96.

Kajava, J., Anttila, J., Varonen, R., Savola, R., Roning, J., (2006). Information Security Standards and Global Business. IEEE International Conference, 2091-2095.

Kremic, T., Tukel, O. I., \& Rom, W. O. (2006). Outsourcing decision support: A survey of benefits, risks, and decision factors. Supply Chain Management. An International Journal, 11(6), 467-482.

Lonsdale, C., \& Cox, A. (2000). The historical development of outsourcing: the latest fad?, Industrial management and data systems, 100(9), 444-450.

Mantel, S.P., Tatikonda, M.V., \& Liao, Y. (2006). A behavioral study of supply manager decision-making: Factors influencing make versus buy evaluation. Journal of Operations Management, 24, 822-838.

McIvor, R. T., \& Humphreys, P. K. (2000). A case-based reasoning approach to the make or buy decision. Integrated Manufacturing Systems Journal, 11(5), 295-310.

McIvor, R. T., Humphreys, P. K., \& McAleer, W. E. (1997). A strategic model for the formulation of an effective make or buy decision. Management Decision, 35(2), 169-78.

Moses, A, \& Ahlstrom, P. (2008). Dimensions of change in make or buy decision processes. Strategic Outsourcing: An International Journal. 1(3), 230-251.

Platts, K.W., Probert, D.R., \& Canez, L. (2002). Make vs. buy decisions: A process incorporating multiattribute decision-making. International Journal of Production Economic, 77, 247-257.

Porter, M., \& Miller, V. (1985). How information gives you competitive advantage. Harvard Business Review, 63(4), 149-160.

Prahalad, C.K., \& Hamel, G. (1990). The core competence of the corporation, Harvard Business Review, 68(3), 79-91.

Probert, D.R. (1997). Developing a Make-or-Buy for Manufacturing Business. Institute of Electrical Engineers, UK.

Probert, D.R., Cáñez, L.E., \& Platts, K.W. (2001). Technology transfer and succession planning: The relevance of make-or-buy analysis. Proceeding of Portland International Conference of Management Engineering. Technology. Portland Oregon, 2, 439-443.

Quinn, J., \& Hilmer, F. (1994). Strategic outsourcing. Sloan Management Review, 35(4):43-55.

Ronan, M. (2010). The influence of capability considerations on the outsourcing decision: the case of a manufacturing company. International Journal of Production Research., 48(17), 5031-5052.

Venkatesan, R. (1992). Strategic sourcing: To make or not to make. Harvard Business Review, 70(6), 98-107.

Wang, T.C., \& Chang, T.H. (2007). Application of TOPSIS in evaluating initial training aircraft under a fuzzy environment. Expert Systems with Applications, 33(4), 870-880.

Wang, J.J., \& Yang, D.L. (2007). Using a hybrid multi-criteria decision aid method for information systems outsourcing. Computers \& Operations Research, 34, 3691-3700.

Water, H., \& Peet, H., (2006). A decision support model based on the Analytic Hierarchy Process for the Make or Buy decision in manufacturing, Journal of Purchasing \& Supply Management, 12, 258-271.

Welch, J., \& Nayak, A. (1992). Strategic sourcing: a progressive approach to the make-or-buy decision. Academic Management Executive, 6(1), 23-31.

Yager, R.R. (1981). A procedure for ordering fuzzy subsets of the unit interval. Information Science, 24, 143161. 